\title{
Clinical utility gene card for: Cornelia de Lange syndrome
}

\author{
Feliciano J Ramos ${ }^{\star 1,2}$, Beatriz Puisac ${ }^{2}$, Carolina Baquero-Montoya ${ }^{3}, \mathrm{M}^{\mathrm{a}}$ Concepción Gil-Rodríguez ${ }^{2}$, \\ Inés Bueno ${ }^{1}$, Matthew A Deardorff ${ }^{4}$, Raoul C Hennekam ${ }^{5}$, Frank J Kaiser ${ }^{6}$, Ian D Krantz ${ }^{7}$, Antonio Musio ${ }^{8}$, \\ Angelo Selicorni ${ }^{9}$, David R FitzPatrick ${ }^{10}$ and Juan Piée ${ }^{\star 2}$
}

European Journal of Human Genetics (2015) 23, doi:10.1038/ejhg.2014.270; published online 24 December 2014

\section{DISEASE CHARACTERISTICS}

1.1 Name of the disease (synonyms)

Cornelia de Lange syndrome (CdLS); Brachmann-de Lange syndrome (BdLS).

\subsection{OMIM\# of the disease}

122470; 300590; 610759; 614701; 300882.

1.3 Name of the analysed genes or DNA/chromosome segments NIPBL; 5p13.2; SMC1A; Xp11.22-p11.21; SMC3;10q25.2; RAD21; 8q24.11;HDAC8; Xq13.1.

1.4 OMIM\# of the gene(s)

${ }^{\star} 608667 ;{ }^{\star} 300040 ;{ }^{*} 606062 ;{ }^{\star} 606462 ;{ }^{\star} 300269$.

\subsection{Mutational spectrum}

CdLS is a congenital autosomal dominant (NIPBL, SMC3 and $R A D 21)$ or X-linked dominant (SMC1A and HDAC8) disorder characterized by facial dysmorphism, pre- and post-natal growth retardation, developmental, intellectual disability, and multiorgan involvement. ${ }^{1,2}$

Currently, it is estimated that $\sim 80 \%$ of patients with CdLS have an identifiable mutation in the NIPBL gene, including the $23 \%$ of cases with somatic mosaicism. ${ }^{3,4}$ More than 300 mutations have been found spreaded throughout the gene, although exon 10 appears to be a hot spot. ${ }^{5}$ Different types of point mutations have been reported, including (in order of decreasing prevalence) frameshift $(32 \%)$, missense $(26 \%)$, nonsense (18\%), splice site $(17 \%)$ and in-frame deletion or insertion (7\%). Moreover, 33 larger deletions and one balanced translocation have been reported as well. A much smaller percentage of patients $(\sim 4-6 \%)$ have mutations in the cohesin complex gene SMC1A, ${ }^{6-8}$ in which 34 mutations have been identified so far, ${ }^{9}$ including missense mutations $(82 \%)$ or in-frame deletions $(18 \%)$. No frameshift or nonsense mutations have been reported in this gene, possibly because they are lethal or lead to a different yet unrecognisable phenotype. ${ }^{5}$

To date, only six patients with mutation in SMC3 have been identified. ${ }^{8,10}$

Mutations in RAD21 have been reported in eight patients with a CdLS phenotype: four whole-gene deletions, two missense, one inframe exonic deletion and one frameshift mutation. ${ }^{11,12}$

Mutations in the HDAC8 gene account for $\sim 4 \%$ of mutations in individuals, including a phenotypically distinct subgroup of CdLS. ${ }^{13,14}$ Reported mutations include missense (the most frequent type), chromosomal microdeletions or microduplications, nonsense and splice site.

HDAC8 mutations have also been found in seven males from the same family affected by intellectual disability, short stature, truncal obesity, craniofacial dysmorphic features, gynaecomastia and hypogonadism, overlapping the X-linked intellectual disability syndromes Wilson-Turner syndrome and Börjeson-Forssman-Lehmann syndrome. ${ }^{15}$

For the standard reference sequence in relation to the variants reported, NCBI Reference Sequences: NM_133433.3 (NIPBL gene), NM_006306.3 (SMC1A), NM_005445.3 (SMC3), NM_006265.2 (RAD21) and NM_018486.2 (HDAC8) should be applied. NIPBL gene variants can be found in the database: Leiden Open Variation Database (LOVD) (http://grenada.lumc.nl/LOVD2/CDLS/ home.php? select_db $=$ NIPBL).

Although most of the identified mutations in the CdLS-related genes are considered de novo, familial cases have been reported in patients with mutations in NIPBL, ${ }^{2,16-19} S M C 1 A,{ }^{7,9} R A D 21^{12}$ and HDAC8. ${ }^{14}$

Although the vast majority of patients with the classic/severe CdLS phenotype carry an identifiable mutation in NIPBL, in some of them

${ }^{1}$ Clinical Genetics Unit, Service of Paediatrics, University Hospital ‘Lozano Blesa' Medical School, University of Zaragoza, CIBERER-GCV and IIS-Aragón, Zaragoza, Spain; ${ }^{2}$ Unit of Clinical Genetics and Functional Genomics, Departments of Pharmacology-Physiology and Paediatrics Medical School, University of Zaragoza, CIBERER-GCV and IIS-Aragón, Zaragoza, Spain; ${ }^{3}$ Department of Paediatrics, Hospital Pablo Tobón Uribe, Medellín, Colombia; ${ }^{4}$ Department of Paediatrics, The Children's Hospital of Philadelphia, Perelman School of Medicine at the University of Pennsylvania, Philadelphia, PA, USA; ${ }^{5}$ Department of Paediatrics, Academic Medical Centre, Amsterdam, The Netherlands; ${ }^{6}$ Sektion für Funktionelle Genetik am Institut für Humangenetik, Universität zu Lübeck, Lübeck, Germany; ${ }^{7}$ Division of Human Genetics, The Children's Hospital of Philadelphia, Perelman School of Medicine at the University of Pennsylvania, Philadelphia, PA, USA; ${ }^{8}$ Istituto di Ricerca Genetica e Biomedica, Consiglio Nazionale delle Ricerche, Pisa, Italy; ${ }^{9}$ Pediatric Genetic Clinic Unit, MBBM Foundation University of Milano Bicocca, San Gerardo Hospital, Monza, Italy; ${ }^{10}$ MRC Human Genetics Unit - MRC IGMM, University of Edinburgh, Edinburgh, UK

*Correspondence: Dr FJ Ramos or Dr J Pié, Unit of Clinical Genetics and Functional Genomics, Departments of Pharmacology-Physiology and Paediatrics, Medical School, University of Zaragoza, CIBERER-GCV and IIS-Aragón, c/ Domingo Miral s/n, E-50009 Zaragoza, Spain. Tel: +34 976 $761739 / 976761677 ;$ Fax: +34 976 761726 ; E-mail: framos@unizar.es or juanpie@unizar.es

Received 12 May 2014; revised 1 November 2014; accepted 16 November 2014; published online 24 December 2014 
no mutation was identified in any of the known CdLS-associated genes. Most of these cases are possibly owing to mosaicism, which in some individuals can be difficult to discern. ${ }^{20}$ It is also hypothesized that other yet unknown gene/s is/are likely involved as well. Moreover, there are individuals with atypical CdLS phenotypes that phenotypically overlap with the classic CdLS phenotype and who are considered to have a 'cohesinopathy'.

\subsection{Analytical methods}

Sequencing of all NIPBL exons and their intron-exon boundaries, multiplex ligation-dependent probe amplification (MLPA) or SNP array/array-CGH to identify large deletions/duplications. On the basis of the type of mutations most frequently identified, start with sequencing, followed by MLPA or SNP array/array-CGH. Although, most of the studies are usually performed using DNA from blood leukocytes, recent publications indicate a high frequency of mosaic NIPBL mutations that are not detected by conventional sequencing of blood DNA. Therefore, the simultaneous study of DNA from buccal cells with highly sensitive technologies (next-generation sequencing (NGS)) or Sanger sequencing of DNA from fibroblasts (skin biopsy) is recommended to improve genetic diagnosis and counselling. ${ }^{21}$ In patients who test negative for mutations in NIPBL, testing of SMC1A, HDAC8, RAD21 and SMC3 should be individually considered.

As soon as the cost of exome sequencing falls to an affordable level and exome coverage increases, panel/whole-exome sequencing will likely become the first-line tier testing technique for this heterogenous disorder.

\subsection{Analytical validation}

Direct sequencing of amplification products is performed in both forward and reverse directions. The existence of a mutation is confirmed by sequencing a second independently amplified PCR product from the patient's DNA from the respective tissue.

RT-PCR and cDNA sequencing are performed to confirm splicing mutations.

1.8 Estimated frequency of the disease (incidence at birth ('birth prevalence') or population prevalence. If known to be variable between ethnic groups, please report)

Birth prevalence: between 1:10000 and 1:30000 live births. No difference between ethnic groups.

\subsection{Diagnostic setting}

\begin{tabular}{lcc}
\hline & Yes & No \\
A. (Differential) diagnostics & $\bigotimes$ & $\square$ \\
B. Predictive testing & $\square$ & $\square$ \\
C. Risk assessment in relatives & $\bigotimes$ & $\square$ \\
D. Prenatal & $\bigotimes$ & $\square$ \\
\hline
\end{tabular}

\section{Comment:}

Predictive testing-not applicable. As CdLS is not a late-onset disease and the clinical features are usually present at birth, the genetic molecular tests, even in young children, are considered diagnostic and not predictive.

Risk assessment is possible in relatives of CdLS individuals with either familial (inherited) or de novo mutations.

\section{TEST CHARACTERISTICS}

\begin{tabular}{|c|c|c|c|c|}
\hline & \multicolumn{2}{|c|}{ Genotype or disease } & \multirow{2}{*}{$\begin{array}{l}\text { A: True positives } \\
\text { B: False positives }\end{array}$} & \multirow{2}{*}{$\begin{array}{l}\text { C: False negative } \\
\text { D: True negative }\end{array}$} \\
\hline & Present & Absent & & \\
\hline \multicolumn{5}{|l|}{ Test } \\
\hline Positive & A & $\mathrm{B}$ & $\begin{array}{l}\text { Sensitivity: } \\
\text { Specificity: }\end{array}$ & $\begin{array}{l}A /(A+C) \\
D /(D+B)\end{array}$ \\
\hline Negative & C & D & $\begin{array}{l}\text { Positive predictive value: } \\
\text { Negative predictive value: }\end{array}$ & $\begin{array}{l}A /(A+B) \\
D /(C+D)\end{array}$ \\
\hline
\end{tabular}

\subsection{Analytical sensitivity}

(proportion of positive tests if the genotype is present)

The most common diagnostic method used for molecular diagnosis of CdLS is Sanger sequencing of DNA extracted from peripheral blood. In patients with mutations in NIPBL and somatic mosaicism analytical sensitivity may vary depending on the analysed sample and on the applied technique. Published studies indicate that analytical sensitivity in blood by Sanger sequencing drops far below 70\% when low-level mosaicism is present. ${ }^{21}$

Mutations outside the coding exons in promoters, regulatory regions or introns will likely be missed as well.

\subsection{Analytical specificity}

(proportion of negative tests if the genotype is not present)

The detection specificity of gene sequencing is $90 \%$ for point mutations, and small deletions and insertions. MLPA or SNP array/ array-CGH are applicable only for exon-spanning mutations and the detection specificity is $95 \%$.

\subsection{Clinical sensitivity}

(proportion of positive tests if the disease is present)

The clinical sensitivity can be dependent on variable factors such as age or family history. In such cases a general statement should be given, even if a quantification can only be made case by case.

On the other hand, it would be difficult to provide a reliable estimate because: a) some patients display a non-classic phenotype, making difficult to ascertain whether they have CdLS (i.e. SMC1Amutated paucisymptomatic females and the not uncommon $H D A C 8$ mutated patients); b) clinical diagnosis may be hard to confirm in patients with low-level mosaic mutations and in patients mutated in yet unknown genes.

\subsection{Clinical specificity}

(proportion of negative tests if the disease is not present)

The clinical specificity can be dependent on variable factors such as age or family history. In such cases a general statement should be given, even if a quantification can only be made case by case.

\subsection{Positive clinical predictive value}

(lifetime risk to develop the disease if the test is positive)

Predictive testing-not applicable, as this syndrome is not a lateonset disease and the clinical features are usually present at birth, molecular-genetic testing must be considered diagnostic and not predictive.

2.6 Negative clinical predictive value (probability not to develop the disease if the test is negative) 
Assume an increased risk based on family history for a non-affected person. Allelic and locus heterogeneity may need to be considered.

Index case in that family had been tested:

Nearly $100 \%$.

Index case in that family had not been tested:

Not a recommended approach.

\section{CLINICAL UTILITY}

3.1 (Differential) diagnostics: The tested person is clinically affected (To be answered if in 1.9 'A' was marked)

\subsubsection{Can a diagnosis be made other than through a genetic test?}

\begin{tabular}{lll}
\hline No & $\square$ (continue with 3.1.4) \\
Yes & $\bigotimes$ & \\
& Clinically & $\square$ \\
Imaging & $\square$ \\
& Endoscopy & $\square$ \\
& Biochemistry & $\square$ \\
& Electrophysiology & $\square$ \\
& Other (please describe)
\end{tabular}

Comment:

Clinical diagnosis of classical CdLS is based on characteristic recognisable facial features (arched eyebrows, synophrys, long eyelashes, short nose with anteverted nares, long and smooth philtrum, thin upper vermillion with downturned corners of the mouth, high palate with widely spaced or absent teeth), limb malformations (short first metacarpal/proximally placed thumb, small hand and/or feet, fifth finger clinodactyly, radial head dislocation/abnormal elbow extension, oligodactyly and reduction defects), growth pattern (pre-postnatal growth retardation with microcephaly) and neurodevelopmental profile (moderate-tosevere global developmental delay, speech and language disabilities, anxiety, obsessive compulsive behaviour with autistic spectrum disorder). Clinical manifestations in patients with mutations in SMC1A and SMC3 typically have a milder phenotype compared with those with NIPBL mutations, particularly regarding limb reduction defects and growth retardation.

Patients with mutations in $R A D 21$ typically show growth retardation, minor skeletal anomalies and facial features that overlap with typical CdLS. Notably, these individuals show a relatively mild phenotype compared with classic CdLS, particularly limb involvement and developmental delay. ${ }^{11,12}$

Patients with mutations in HDAC8 have features suggestive of CdLS but with some atypical features including large anterior fontanel, hooded eyelids, broader nasal root and pleasant personality. ${ }^{13}$ In this $\mathrm{X}$-linked form of CdLS the hemizygous males are more severely affected. In females the severity is strongly influenced by random $\mathrm{X}$ inactivation. ${ }^{14}$ HDAC8 mutations have also been found in an X-linked intellectual disability syndrome which overlaps Wilson-Turner syndrome and Börjeson-Forssman-Lehmann syndrome. ${ }^{15}$

CdLS has been reported to have some clinical overlap with Fryns syndrome (coarse face, diaphragmatic hernia, cleft palate, distal limb hypoplasia and hypertrichosis), ${ }^{22}$ foetal alcohol syndrome (pre-postnatal growth retardation, developmental delay, hirsutism, craniofacial anomalies and cardiac defects), ${ }^{23}$ and Coffin-Siris Syndrome (aplasia or hypoplasia of the distal phalanx or nail of the fifth digit, distinctive facial features and moderate-to-severe developmental delay). ${ }^{24,25}$

3.1.2 Describe the burden of alternative diagnostic methods to the patient

The burden is minimal as clinical features are often sufficient to make a definitive diagnosis.

\subsubsection{How is the cost effectiveness of alternative diagnostic methods to be judged?}

Clinical diagnosis is a relatively inexpensive procedure. Genetic testing of the five CdLS associated genes is still relatively expensive, although the use of panel-sequencing methodology has lowered the cost. Moreover, the availability of the new molecular diagnostic techniques of nextgeneration sequencing (NGS), whole-genome/exome sequencing will significantly improve the mutation rate detection at an affordable cost.

\subsubsection{Will disease management be influenced by the result of a} genetic test?

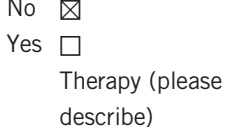
describe)

Management (please
describe)

3.2 Predictive Setting: The tested person is clinically unaffected but carries an increased risk based on family history

(To be answered if in 1.9 'B' was marked)

\subsubsection{Will the result of a genetic test influence lifestyle and prevention?}

If the test result is positive (please describe)

Not applicable.

If the test result is negative (please describe)

Not applicable.

3.2.2 Which options in view of lifestyle and prevention does a person at-risk have if no genetic test has been done (please describe)? Not applicable.

3.3 Genetic risk assessment in family members of a diseased person (To be answered if in 1.9 ' $\mathrm{C}$ ' was marked)

\subsubsection{Does the result of a genetic test resolve the genetic situation in that family?}

NIPBL, SMC3 and RAD21-related CdLS mutations are inherited in an autosomal dominant manner. The majority of affected individuals 
have a de novo NIPBL mutation; fewer than $2 \%$ of individuals with NIPBL-related CdLS have an affected parent. When both parents are clinically unaffected, the risk of the sibs of a proband with NIPBLrelated CdLS is estimated to be 3-5\% (slightly higher than general population) because of the possibility of germline mosaicism. ${ }^{3,19,26}$ $S M C 1 A$ and HDAC8 are inherited in an X-linked dominant manner although SMC1A partially escapes X inactivation, whereas HDAC8 is subject to $\mathrm{X}$ inactivation. The risk of the sibs of a proband with SMC1A/HDAC8-related CdLS depends on the status of the proband's mother. Prenatal testing for pregnancies at increased risk is possible for families in which the disease-causing allele has been identified.

As CdLS is an autosomal dominant/X-linked disease with almost complete penetrance and high intra-familial phenotypic variability, affected individuals and their relatives should be offered genetic counselling and testing. There is an exception in the case of HDAC8 female carriers inactivating the mutant allele, making relevant their recognition to evaluate the risk for proband's sibs.

\subsubsection{Can a genetic test in the index patient save genetic or other test in family members?}

Not completely. However, identification of the causative mutation in the index case allows focussed genetic testing in family members instead of screening of the entire gene or of all genes potentially causing CdLS. If the mutation in the proband is found to be de novo (e.g. not present in the parents) then no one else in the extended family needs to be tested. As there is a significant amount of germline mosaicism reported in CdLS it may still be relevant to offer prenatal testing to affected families with one child with CdLS, even when the mutation is not identified in the blood of the parents.

\subsubsection{Does a positive genetic test result in the index patient enable a predictive test in a family member?}

See comment in 1.9 'B'.

\subsection{Prenatal diagnosis}

(To be answered if in 1.9 'D' was marked)

\subsubsection{Does a positive genetic test result in the index patient enable a prenatal diagnosis?}

Possible if mutation is identified in any of the known causative genes.

\section{IF APPLICABLE, FURTHER CONSEQUENCES OF TESTING}

Please assume that the result of a genetic test has no immediate medical consequences. Is there any evidence that a genetic test is nevertheless useful for the patient or his/her relatives?

The result of a CdLS genetic test may have no immediate medical consequences for the affected individuals and their families, but having a positive genetic diagnosis will influence genetic counselling and may have some impact in reproductive decisions.

\section{CONFLICT OF INTEREST}

The authors declare no conflict of interest.

\section{ACKNOWLEDGEMENTS}

This work was supported by EuroGentest2 (Unit 2: 'Genetic testing as part of health care'), a Coordination Action under FP7 (Grant Agreement Number 261469) and the European Society of Human Genetics. FJR and JP are supported by a grant from the Spain's Ministry of Health-ISCIII (Ref. FIS PI12 01318) and the Gobierno de Aragón (Ref. B20). FJK is supported by the German Federal Ministry of Education and Research (BMBF) in frame of
E-Rare-2 (TARGET-CdLS). P01 HD052860 (from the National Institutes of Health $(\mathrm{NOH}) / \mathrm{National}$ Institute of Child Health and Development (NICHD), USA to IDK).

1 Tonkin ET, Smith M, Eichhorn $\mathrm{P}$ et al: A giant novel gene undergoing extensive alternative splicing is severed by a Cornelia de Lange-associated translocation breakpoint at 3q26.3. Hum Genet 2004; 115: 139-148.

2 Krantz ID, McCallum J, DeScipio C et al: Cornelia de Lange syndrome is caused by mutations in NIPBL, the human homolog of Drosophila melanogaster Nipped-B. Nat Genet 2004; 36: 631-635.

3 Huisman SA, Redeker EJ, Maas SM, Mannens MM, Hennekam RC: High rate of mosaicism in individuals with Cornelia de Lange syndrome. J Med Genet 2013; 50 339-344

4 Castronovo P, Delahaye-Duriez A, Gervasini C et al: Somatic mosaicism in Cornelia de Lange syndrome: a further contributor to the wide clinical expressivity? Clin Genet 2010; 78: 560-564.

5 Mannini L, Cucco F, Quarantotti V, Krantz ID, Musio A: Mutation spectrum and genotype-phenotype correlation in Cornelia de Lange syndrome. Hum Mutat 2013; 34: 1589-1596.

6 Musio A, Selicorni A, Focarelli ML et al: X-linked Cornelia de Lange syndrome owing to SMC1L1 mutations. Nat Genet 2006; 38: 528-530.

7 Revenkova E, Focarelli ML, Susani L et al: Cornelia de Lange syndrome mutations in SMC1A or SMC3 affect binding to DNA. Hum Mol Genet 2009; 18: 418-427.

8 Deardorff MA, Kaur M, Yaeger D et al: Mutations in cohesin complex members SMC3 and SMC1A cause a mild variant of Cornelia de Lange syndrome with predominant mental retardation. Am J Hum Genet 2007; 80: 485-494.

9 Gervasini C, Russo S, Cereda A et al: Cornelia de Lange individuals with new and recurrent SMC1A mutations enhance delineation of mutation repertoire and phenotypic spectrum. Am J Med Genet A 2013; 161A: 2909-2919.

10 Ansari M, Poke G, Ferry Q et al: Genetic heterogeneity in Cornelia de Lange syndrome (CdLS) and CdLS-like phenotypes with observed and predicted levels of mosaicism. J Med Genet 2014; 51: 659-668.

11 Deardorff MA, Wilde JJ, Albrecht $\mathrm{M}$ et al: RAD21 mutations cause a human cohesinopathy. Am J Hum Genet 2012; 90: 1014-1027.

12 Minor A, Shinawi M, Hogue J et al: Two novel RAD21 mutations in patients with mild Cornelia de Lange syndrome-like presentation and report of the first familial case. Gene 2014; 537: 279-284.

13 Deardorff MA, Bando M, Nakato R et al: HDAC8 mutations in Cornelia de Lange syndrome affect the cohesin acetylation cycle. Nature 2012; 489: 313-317.

14 Kaiser FJ, Ansari M, Braunholz D et al: Loss of Function HDAC8 Mutations Cause a Phenotypic Spectrum of Cornelia de Lange Syndrome-like Features, Ocular Hypertelorism, Large Fontanelle and X-linked Inheritance. Hum Mol Genet 2014; 23 2888-2900.

15 Harakalova M, van den Boogaard MJ, Sinke R et al: X-exome sequencing identifies a HDAC8 variant in a large pedigree with X-linked intellectual disability, truncal obesity, gynaecomastia, hypogonadism and unusual face. J Med Genet 2012; 49: 539-543.

16 Gillis LA, McCallum J, Kaur M et al: NIPBL mutational analysis in 120 individuals with Cornelia de Lange syndrome and evaluation of genotype-phenotype correlations. Am J Hum Genet 2004; 75: 610-623.

17 Borck G, Zarhrate M, Cluzeau C et al: Father-to-daughter transmission of Cornelia de Lange syndrome caused by a mutation in the $5^{\prime}$ untranslated region of the NIPBL Gene. Hum Mutat 2006; 27: 731-735.

18 Niu DM, Huang JY, Li HY et al: Paternal gonadal mosaicism of NIPBL mutation in a father of siblings with Cornelia de Lange syndrome. Prenat Diagn 2006; 26 1054-1057.

19 Weichert J, Schröer A, Beyer DA, Gillessen-Kaesbach G, Stefanova I: Cornelia de Lange syndrome: antenatal diagnosis in two consecutive pregnancies due to rare gonadal mosaicism of NIPBL gene mutation. J Matern Fetal Neonatal Med 2011; 24: 978-982.

20 Slavin TP, Lazebnik N, Clark DM et al: Germline mosaicism in Cornelia de Lange syndrome. Am J Med Genet A 2012; 158: 1481-1485.

21 Braunholz D, Obieglo C, Parenti I et al: Hidden mutations in CdLS-Limitations of Sanger Sequencing in Molecular Diagnostics. Hum Mutat 2014; doi:10.1002/humu. 22685 [Epub ahead of print]

22 Slavotinek AM.: Fryns syndrome: a review of the phenotype and diagnostic guidelines. Am J Med Genet A 2004; 124A: 427-433.

23 Douzgou S, Breen C, Crow YJ et al: Diagnosing fetal alcohol syndrome: new insights from newer genetic technologies. Arch Dis Child 2012; 97: 812-817.

24 Schrier SA, Bodurtha JN, Burton B et al: The Coffin-Siris syndrome: a proposed diagnostic approach and assessment of 15 overlapping cases. Am J Med Genet $A$ 2012; 158A: 1865-1876.

25 Santen GW, Aten E, Vulto-van Silfhout AT et al: Coffin-Siris syndrome and the BAF complex: genotype-phenotype study in 63 patients. Hum Mutat 2013; 34 1519-1528.

26 Mariani M, Bettini LR, Cereda A et al: Germline mosaicism in Cornelia de Lange syndrome: dilemmas and risk figures. Am J Med Genet A 2013; 161A: 1825-1826. 